\section{AB0240 ALCOHOL CONSUMPTION AND DEVELOPMENT OF ARTHRITIS AMONG PATIENTS WITH ANTI- CITRULLINATED PEPTIDE ANTIBODIES AND MUSCULOSKELETAL PAIN}

Emma Eloff ${ }^{1}$, Michael Ziegelasch ${ }^{1}$, Klara Martinsson ${ }^{2}$, Jan Cedergren ${ }^{1}$, Åsa Reckner ${ }^{1}$, Thomas Skogh ${ }^{1}$, Louise Karlsson ${ }^{3}$, Andreas Ärlemalm $^{3}$, Alf Kastbom ${ }^{1} .{ }^{1}$ Department of Rheumatology, Linköping, Sweden; ${ }^{2}$ Department of Clinical and Experimental Medicine, Linköping, Sweden; ${ }^{3}$ Department of Clinical Pharmacology, Linköping, Sweden

Background: Individuals with anti-citrullinated peptide antibodies (ACPA) and arthralgia are at increased risk of developing rheumatoid arthritis (RA). Predictors of disease development are important within this category of patients in order to improve treatment and follow-up decisions. Although excessive use of alcohol is well-known to cause harmful medical and social consequences, an inverse association between alcohol consumption and RA development has been proposed. Phosphatidylethanol (PEth) has shown to be a reliable biomarker to measure recent (up to four weeks) alcohol consumption with high specificity.

Objectives: The aim of this study was to, in relation to other possible clinical and laboratory predictors, pinpoint the association between biochemically determined alcohol consumption and development of arthritis in ACPA-positive individuals with musculoskeletal pain.

Methods: The study was performed as part of an observational prospective cohort (TIRx), including 104 ACPA-positive individuals with musculoskeletal pain and maximally one arthritis upon clinical examination. Exclusion criteria were $>1$ clinical arthritis, previous inflammatory rheumatic disease, and oral or intraarticular corticosteroid treatment within 6 weeks prior to screening. Participants were enrolled between 2010 and 2013 and were carefully followed during 72 months in median (range 23-91). The primary outcome measure was development of arthritis upon clinical examination. In baseline samples, we assessed ACPA levels in serum $\left(2^{\text {nd }}\right.$ generation cyclic citrullinated peptides (CCP) as antigen), rheumatoid factor (RF), and the presence of shared epitope. PEth 16:0/18:1 was measured by liquid chromatography coupled with tandem mass spectrometry (LC-MS/MS) in whole blood from baseline, and the results were categorized into three groups: no/low, moderate, or high consumption. Cox regression analyses were performed adjusting for smoking, symptom duration, age, sex, shared epitope, RF, and treatment with disease modifying antirheumatic drugs (DMARDs) and oral glucocorticoids.

Results: In TIRx, 82 patients had no swollen joints at inclusion, of whom $39(48 \%)$ developed arthritis during follow-up after median 6 months (range 1-71). Of those, 48 (59\%) patients were classified according to PEth values with no/low alcohol consumption, 28 (34\%) with moderate consumption and $5(6 \%)$ patients with high alcohol consumption. There was no significant difference in PEth values between patients with one baseline arthritis compared to those without $(p=0.09)$. Neither were there any significant differences in arthritis-free survival across PEth categories versus arthritis development $(p=0.64)$. Unadjusted hazard ratios (HRs) were numerically, but not significantly, increased among moderate (HR $1.2295 \% \mathrm{Cl}$ 0.63-2.37, $\mathrm{p}=0.56$ ) and high consumers (HR $1.6995 \% \mathrm{Cl}$ $0.50-5.68, p=0.40$ ) as compared to those classified as no/low consumers. There was an increased risk of arthritis development regarding RF positivity (adjusted HR 3.13, 95\% Cl 1.36-7.19, $\mathrm{p}=0.007$ ) and higher ACPA levels (adjusted HR 1,001 95\% Cl 1.000-1.001, $\mathrm{p}<0.001$ ), respectively. Conclusion: This study does not show a significant association between biochemically assessed recent alcohol consumption and arthritis development in ACPA-positive individuals with musculoskeletal pain. Thus, PEth does not appear to be a clinically useful biomarker to predict disease development in ACPA-positive at-risk populations. Whether it may predict arthritis in a seronegative population remains to be determined. We confirm that RF positivity and ACPA levels associate with arthritis development in an ACPA-positive population.

Disclosure of Interests: Emma Eloff: None declared, Michael Ziegelasch Consultant for: AbbVie, MSD, Pfizer, and BK-Medical, Klara Martinsson: None declared, Jan Cedergren: None declared, Åsa Reckner: None declared, Thomas Skogh: None declared, Louise Karlsson: None declared, Andreas Ärlemalm: None declared, Alf Kastbom Consultant for: Roche and Pfizer, Employee of: Sanofi, Speakers bureau: UCB and BMS DOI: 10.1136/annrheumdis-2019-eular.1644

\section{AB0241 DISEASE COURSE AND COSTS OF A COHORT OF RHEUMATOID ARTHRITIS PATIENTS OVER A PERIOD OF 6 YEARS}

Gizem Ayan, Sinem Nihal Esatoglu, Gulen Hatemi, Vedat Hamuryudan. Istanbul University-Cerrahpasa, Cerrahpasa Medical Faculy, Department of Internal Medicine, Division of Rheumatology, Istanbul, Turkey

Background: A previous multicenter, cross-sectional study showed a substantial economic burden of rheumatoid arthritis (RA) in Turkey with significant positive correlation between disease severity and total costs (1). Objectives: To calculate the annual costs and to assess disease activity and functional outcome of RA patients from a single center over a period of 6 years.

Methods: The previous study on healthcare costs in RA was performed in the outpatient clinics of 10 university hospitals between May 2011 and August 2012 (1). A total of 689 RA patients (all fulfilling ACR 1987 criteria) were studied of whom $75(11 \%)$ being from our center. In March 2018, we called back our patients to the clinic for re-evaluation by using the questionnaire of the previous study containing questions on demographics, medication use and RA-related direct and indirect costs. We assessed disease activity with the Routine assessment of Patient Index Data 3 (RAPID3), functional status with $\mathrm{HAQ}-\mathrm{DI}$ and Steinbrocker functional index and quality of life (QoL) with EuroQol Quality of Life Scale (EQ-5D).

Results: We could interview 62 patients (83\%) in the clinic. Of the remaining 13 patients, $7(9 \%)$ had died, 3 were receiving palliative care following cardio-vascular events, 2 went to other centers and 1 declined to participate. The mean age and mean disease duration of the 62 reevaluated patients (52 women, 10 men) were $56.8 \pm 13.3$ SD years and $225 \pm 120 \mathrm{SD}$ months, respectively. Forty-nine $(79 \%)$ had used at least 1 biologic agent during follow-up and 34 (55\%) were still on biologics at the time of re-evaluation. Disease activity was lower but was not significantly different from that of the previous study. However, functional status and QoL had improved significantly over time (Table 1). The majority of the patients $(89 \%)$ were in Steinbrocker Class 1 or 2 with only $7(11 \%)$ being in Steinbrocker Class 3. Of the 7 deceased patients (4 women 3 men; mean age: $68.2 \pm 8.12 \mathrm{SD}$ years; mean disease duration: $127.6 \pm 73.8$ SD months) 3 were on Rituximab, 2 were on synthetic DMARD's (one being biologic naïve) and 2 were free of RA medications (one was biologic naïve) at the time of death. Serious infections were the cause of death in 4 patients followed by hepatic failure due to hepatitis B, abdominal bleeding under anticoagulation and multi organ failure in 3 patients, respectively. Direct costs were higher than indirect costs and made up two thirds of RA related total costs (Table 1).

Conclusion: Disease activity remained stable and functional status and QoL improved among our patients over 6 years. Biologic usage was increased. Cardiovascular events and serious infections were major determinants of morbidity and mortality. Direct costs were the main determinants of RA related cost.

Table 1:. RAPID 3, HAQ-DI, EQD5 Scores and annual cost

\begin{tabular}{lccc}
\hline & $\begin{array}{c}\text { Previous } \\
\text { study } \\
(\mathrm{n}=62)\end{array}$ & $\begin{array}{c}\text { Current } \\
\text { study } \\
(\mathrm{n}=62)\end{array}$ & $P$ \\
\hline Mean (SD) RAPID3 Score & $4.85 \pm 1.64$ & $4.36 \pm 1.54$ & 0.06 \\
Mean (SD) HAQ-DI Score & $1.86 \pm 0.59$ & $0.69 \pm 0.57$ & 0.001 \\
Mean (SD) EQ-5D Score & $0.57 \pm 0.21$ & $0.68 \pm 0.21$ & 0.003 \\
Annual mean (SD) direct costs $(€)$ & $\mathrm{NA}^{*}$ & $2262 \pm 2227$ & \\
Annual mean (SD) indirect costs & $\mathrm{NA}^{*}$ & $1220 \pm 2449$ & \\
$(€)$ & & & \\
\hline
\end{tabular}

Results: The survey was replied by 41 rheumatologists, representing all regions in the country. Table 1 shows a summary of the results of the second round in terms of $\mathrm{m}$ and $\mathrm{SD}$. To summarize, there was an agreement regarding the drugs that might be more adequate for patients with particular prognostic factors, except in the case of pulmonary involvement, in which agreement was only met for T-cell co-stimulation, and for elevated $\mathrm{HAQ}$ and acute phase reactants, where the use of Bcell depressant treatments did not reach an $80 \%$ of agreement. $\mathrm{NA}^{*}=$ not available 
REFERENCE:

[1] Hamuryudan V, et al. Clin Exp Rheumatol 2016; 34: 1033

Acknowledgement Supported with an unrestricted grant from Pfizer Disclosure of Interests: Gizem Ayan: None declared, Sinem Nihal Esatoglu: None declared, Gulen Hatemi Consultant for: Abbvie, Amgen, BMS, Janssen, MSD, Pfizer, UCB, Speakers bureau: Abbvie, Amgen, BMS, Jansen, MSD, Pfizer, UCB, Vedat Hamuryudan Consultant for: Abbvie, Amgen, BMS, Jansen, MSD, Pfizer, UCB, Speakers bureau: Abbvie, Amgen, BMS, Jansen, MSD, Pfizer, UCB,

DOI: 10.1136/annrheumdis-2019-eular.984

\section{AB0242 RADIOLOGICAL EVALUATION OF FOREFOOT INVOLVEMENT IN RHEUMATOID ARTHRITIS}

Rym Fakhfakh, Saoussen Zrour, Abir Dghaies, Hibatallah Mosbeh, Olfa Jmaa, Grassa Rim, Jguirim Mahbouba, Ismail Bejia, Mongi Touzi, Naceur Bergaoui. CHU Fattouma Bourguiba, Rheumatology, monastir, Tunisia

Background: Severe and often debilitating involvement of the forefoot is seen frequently in patients with rheumatoid arthritis (RA)

Objectives: We aim to determine the prevalence and the characteristics of radiological forefoot involvement

Methods: Monocentric retrospective study of 52 consecutive patients with RA, hospitalized in the Rheumatology Department between 2012 and 2018. Radiological measurements included the modified Sharp/van der Heijde method (SHS) for the feet and forefoot angles: The angle between the longitudinal bisection of the first metatarsal and the first phalanx of the first finger (Hallux valgus: $\mathrm{H} 1 \mathrm{M} 1 \geq 20^{\circ}$ ), the intermetatarsal angle between the 1st and 2nd metatarsals (Metatarsus primus varus: $M 1 M 2 \geq 10^{\circ}$ ) and the intermetatarsal angle between the 1st and 5th metatarsals (splaying foot: $\mathrm{M} 1 \mathrm{M} 5 \geq 35^{\circ}$ ) measured on antéroposterior radiographs.

Results: The average age was $53 \pm 15$ years and the sex-ratio was 0.21 . The average disease duration was $8 \pm 10$ years and the average disease activity index DAS28 was $5.65 \pm 1.12$. The prevalence of forefoot erosion and joint space narrowing were $35 \%$ and $58 \%$, respectively. In $85 \%$ of patients, both $\geq 1$ forefoot erosion score and $\geq 1$ forefoot joint space narrowing score were present. Erosions were the most frequent in the $5^{\text {th }}$ metatarsophalangeal joints (MTP) and $1^{\text {st }}$ interphalangeal joint $(50 \%$ and $47 \%$ of eroded joints, respectively) and the least frequent in $4^{\text {th }}$ MTP $(27 \%)$. The forefoot erosion score was significantly correlated with age, disease duration and hand erosion score and weakly but statistically significantly with titer of ACPA $(\mathrm{p} \leq 0.05)$. Obesity was an independent protective factor $(\mathrm{OR}=0.25 ; p<0.05)$ of forefoot erosions. Foot deformities were observed in $71 \%$ of the patients; Metatarsus primus varus(MV) $(56 \%)$, hallux valgus(HV) (38.5\%) and splaying foot(SF) (13.5\%). The HV was significantly correlated with forefoot damage (erosion score $r=0.67$, joint space narrowing $r=0.7 ; p<0.01$ ), extra-articular manifestations of $R A$, disease duration and rheumatoid factor titer. Multivariate analysis showed that the extra-articular manifestations was an independent risk factor for HV $(O R=9.39, p<0.05)$. There was significant correlation between the $\mathrm{MV}$ and forefoot damage (erosion score: $\mathrm{r}=0.4$, joint space narrowing: $r=0.5, p<0.05)$. There was no correlation between the splaying foot and the forefoot damage. The splaying foot was associated with the hallux valgus $(\mathrm{OR}=5.7, \mathrm{p}=0.05)$.

Conclusion: In our study, forefoot involvement is common in patients with RA and it isn't associated with disease severity. Deformities (HV, $\mathrm{MV}$ ) are associated with forefoot joint damage and splaying foot is associated with hallux valgus.

\section{REFERENCES:}

[1] Baan H, \& Drossaers-Bakker w, et al. We should not forget the foot: relations between signs and symptoms, damage, and function in rheumatoid arthritis. Clin Rheumatol (2011) 30:1475-1479

[2] Matsumoto, et al. Radiologic Patterning of Joint Damage to the Foot in Rheumatoid Arthritis. Arthritis Care \& Research.2014;66 (4):499-507.

Disclosure of Interests: None declared

DOI: 10.1136/annrheumdis-2019-eular.6967
$\mathrm{AB} 0243$

CYSTEINE RICH 61 (CYR61): A POTENTIAL BIOMARKER ASSOCIATED WITH DISEASE ACTIVITY IN RHEUMATOID ARTHRITIS

Yong Fan, Xinlei Yang, Juan Zhao, Xiaoying Sun, Wenhui Xie, Yanrong Huang, LI Guangtao, Yanjie Hao, Zhuoli Zhang. Peking University First Hospital, Department of Rheumatology and Clinical Immunology, Beijing, China

Background: Numerous preclinical studies have revealed a critical role of Cysteine rich 61 (Cyr61) in the pathogenesis of rheumatoid arthritis (RA). However, little is known about the potential value of circulation Cyr61 in clinical RA patients.

Objectives: To compare serum Cyr61 level in patients with RA and healthy controls, and to characterize the potential association between Cyr61 and RA disease activity.

Methods: In training cohort, serum samples were obtained from 177 patients with definite RA and 50 age- and gender- matched healthy controls. Medical records were collected and serum Cyr61 concentration was detected by enzyme-linked immunosorbent assay. Correlations between Cyr61 levels with clinical disease activity were analyzed. Furthermore, a validation cohort consisting of 77 active RA patients who received uniform biologic therapy for 12 weeks was set up (ClinicalTrials.gov identifier: NCT02320630). Paired serum samples were collected at baseline and after 12-week treatment for each individual and prepared for detection of Cyr61. Comparisons between groups were made using Mann-Whitney $U$ test or Wilcoxon matched-pairs signed rank test, as appropriate.

Results: Significant elevation of serum Cyr61 concentration was found in RA patients, demonstrating excellent diagnostic ability to discriminate RA with healthy controls (area under the curve $(A \cup C)=0.98, P<0.001$ ). In training cohort, Cyr61 level in active RA patients was significantly lower than that in inactive RA, and it was inversely with measures of clinical disease activity in statistic. Findings were further confirmed in our validation cohort. Active RA patients who had a reduction in disease activity showed a significantly increase of Cyr61 level after effective treatment (in terms of achieving ACR20/50/70 improvement criteria). RA patients who did not achieve ACR response showed no significant difference of Cyr61 level before and after treatment. Multivariate logistic regression analysis revealed that increase of Cyr61 level as well as younger age were independent indicators for achieving ACR20 response.

Conclusion: Serum Cyr61 levels were remarkably increased in RA patients compared with healthy control. More intriguingly, the level of Cyr61 was inversely associated with RA disease activity and increased after effective treatment.

\section{REFERENCES:}

[1] Zhang Q, Wu J, Cao Q, Xiao L, Wang L, He D, et al. A critical role of Cyr61 in interleukin-17-dependent proliferation of fibroblast-like synoviocytes in rheumatoid arthritis. Arthritis \& Rheumatism. 2009;60(12):3602-12.

[2] Chen CY, Su CM, Hsu CJ, Huang CC, Wang SW, Liu SC, et al. CCN1 Promotes VEGF Production in Osteoblasts and Induces Endothelial Progenitor Cell Angiogenesis by Inhibiting miR-126 Expression in Rheumatoid Arthritis. J BONE MINER RES. 2017 2017-01-01;32(1):34-45.

[3] Barranco C. CCN1, a novel RA target? NAT REV RHEUMATOL. 2016 2016-08-19;12(10):561

Conflict of interest: The authors declare that they have no competing interests.

Disclosure of Interests: None declared

DOI: 10.1136/annrheumdis-2019-eular.5222

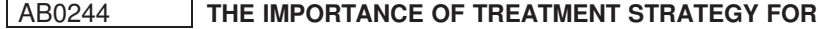 THE OUTCOME OF EARLY RHEUMATOID ARTHRITIS PATIENTS NOT RESPONDERS TO THE FIRST LINE THERAPY WITH METHOTREXATE}

Anna Laura Fedele, Dario Bruno, Barbara Tolusso, Luca Petricca, Gianfranco Ferraccioli, Elisa Gremese. Fondazione Policlinico Universitario $A$. Gemelli IRCCS, Division of Rheumatology, Rome, Italy

Background: According to EULAR recommendations for the management of Rheumatoid Arthritis (RA), if the treatment target is not reached with the first conventional synthetic (cs)-disease modifying antirheumatic drugs (DMARDs), addition of another csDMARD or of a biological (b)-DMARD should be considered [1].

Objectives: To evaluate the clinical and radiological outcome under combination therapy of either cs-DMARDs or b-DMARDs in our cohort of early rheumatoid arthritis (ERA) patients treated according to the treat-totarget (T2T) strategy. 